\title{
The effectiveness of supplemental oxygen during exercise training in patients with chronic obstructive pulmonary disease who show sever exercise-induced desaturation: a protocol for a meta-regression analysis and systematic review
}

Shohei Kawachi

Shinshu Daigaku https://orcid.org/0000-0002-5093-5125

Shuhei Yamamoto

Shinshu Daigaku Igakubu Fuzoku Byoin

Kenichi Nishie

Shinshu Daigaku

\section{Takayoshi Yamaga}

Health science university, Yamanashi, Japan

Manaka Shibuya

Kitasato Daigaku Byoin

Yasunari Sakai

Shinshu Daigaku Igakubu Fuzoku Byoin

Keisaku Fujimoto ( $\square$ keisaku@shinshu-u.ac.jp )

Department of Clinical Laboratory Sciences, Shinshu University School of Health Sciences, Japan https://orcid.org/0000-0002-6512-1757

\section{Protocol}

Keywords: supplemental oxygen, desaturation, hypoxemia, chronic obstructive pulmonary disease, exercise training

Posted Date: May 11th, 2020

DOI: https://doi.org/10.21203/rs.3.rs-26926/v1

License: (1) This work is licensed under a Creative Commons Attribution 4.0 International License.

Read Full License 
Version of Record: A version of this preprint was published at Systematic Reviews on April 14th, 2021. See the published version at https://doi.org/10.1186/s13643-021-01667-9. 


\section{Abstract}

Background: Supplemental oxygen during exercise training has been used to prevent hypoxemia or allow toleration of higher intensity training in patients with chronic obstructive pulmonary disease (COPD) who show exercise-induced desaturation. Although some meta-analyses examined the efficacy of supplemental oxygen during exercise training, these studies concluded that it does not further improve exercise tolerance compared to exercise training alone. However, supplemental oxygen during exercise training may be effective to improve exercise tolerance in COPD patients with severe desaturation during exercise under breathing room air or compressed air. Therefore, this study will be performed to elucidate the efficacy of supplemental oxygen during exercise training and the relationship between its efficacy and severity of desaturation during exercise at baseline.

Methods: We will first assess the effectiveness of supplemental oxygen during exercise training in COPD. The main outcome is the change in exercise tolerance before and after the intervention, indicated by 6minute walking distance or shuttle walking distance, and analyzed as the standardized mean difference (SMD). The quality and risk of bias in individual studies will be assessed using the Grading of Recommendations Assessment, Development, and Evaluation (GRADE) system and risk-of-bias tool (RoB ver.2). If statistical heterogeneity in effectiveness of exercise tolerance is shown, we will conduct metaregression analyses to examine the association between efficacy of exercise training with supplemental oxygen and severity of desaturation at baseline.

Discussion: One strength of this study is that it is a systematic review with meta-regression analysis to elucidate the effectiveness of supplemental oxygen during exercise training in patients with COPD who show sever exercise-induced desaturation. However, the quality and number of studies included metaanalysis may be low.

Systematic review registration: Registration number, UMIN000039960.

\section{Description Of The Condition}

Chronic obstructive pulmonary disease (COPD) is a preventable and treatable disease characterized by persistent respiratory symptoms and airflow limitation due to airway and alveolar abnormalities caused by exposure to harmful particles or gases. ${ }^{1}$ An epidemiological study estimated that COPD has an incidence of 384 million in 2020 , with a prevalence of $11.7 \%$ worldwide. ${ }^{2}$ Globally, 3 million people die from COPD annually.3

As it have been reported that exercise training in rehabilitation improves exercise tolerance, which is related to mortality in COPD, ${ }^{4}$ the Global Initiative for Chronic Obstructive Lung Disease (GOLD) criteria recommend rehabilitation regardless of severity of COPD. ${ }^{1}$ However, as COPD patients who show exercise-induced desaturation are not able to tolerate high-intensity training, its effectiveness in such cases is limited. In COPD, exercise-induced desaturation is caused by decreases of diffusion capacity and 
ventilation perfusion mismatch due to alveolar destruction and airflow obstruction, respectively. ${ }^{5,6}$ Furthermore, patients with severe COPD have more severe exercise-induced desaturation as a result of worsening alveolar hypoventilation by dynamic lung hyperinflation related to airflow limitation on exertion. ${ }^{7,8}$ As exercise-induced desaturation is involved in adverse sequelae and higher risk of mortality in COPD, ${ }^{9,10}$ supplemental oxygen during exercise training has been prescribed in COPD patients who show severe desaturation.

The efficiency of combined supplemental oxygen and exercise training remains unclear. Previous studies showed a twofold increase in exercise training load in the supplemental oxygen group compared to the control group, and therefore the supplemental oxygen group also showed improvements in exercise tolerance, dyspnea, and depressive symptoms. ${ }^{11,12}$ However, other studies failed to show significant improvements in exercise tolerance with supplemental oxygen. ${ }^{13,14}$ In 2007 and 2019, meta-analyses were conducted to elucidate the efficacy of supplemental oxygen during exercise training. ${ }^{15,16}$ The metaanalysis in 2019 concluded that supplemental oxygen during exercise training does not further improve exercise tolerance compared to exercise training alone. ${ }^{16}$ However, especially in COPD patients with severe desaturation at rest and on exertion, supplemental oxygen during exercise training may be effective to improve exercise tolerance. A previous study reported that patients with desaturation by more than $4 \%$ and to less than $90 \%$ on exertion, and who walked $10 \%$ or more further with ambulatory oxygen on endurance shuttle walk test showed significant improvements in exercise tolerance by a 6 - 7-week exercise training program with supplemental oxygen. ${ }^{17}$ In moderate to severe COPD patients with $\mathrm{SpO}_{2}$ during exercise $\geq 90 \%$, endurance training three times/week resulted in significant improvements in quality of life (QOL) and exercise capacity independent of oxygen supplementation, and there were no further benefits of supplemental oxygen from 12 to 24 weeks despite progressively increased training loads. ${ }^{18}$ Therefore, we will also conduct meta-regression analyses to examine the association between efficacy of exercise training with supplemental oxygen and severity of desaturation under breathing room air at baseline.

\section{Objectives}

The objective of this systematic review and meta regression analysis is to elucidate the efficacy of supplemental oxygen during exercise training and the relationship between its efficacy and severity of desaturation in COPD.

\section{Methods And Analyses}

This systematic review will be conducted according to the Cochrane Handbook for Systematic Review of Interventions, the Preferred Reporting Items for Systematic Reviews, Meta-Analyses (PRISMA) statement. ${ }^{19,20}$ The Grading of Recommendations Assessment, Development, and Evaluation (GRADE) system will be used to assess the evidence in cumulative evidence. ${ }^{21}$ This protocol paper was prepared according to PRISMA-P checklist which was uploaded as a supplementary material. ${ }^{22}$ 


\section{Type of study}

Randomized controlled trials (RCTs) regardless of cluster or individual randomization between January 1 , 1980 and May 31, 2020 will be included restricted to those published in English. In addition, full-text studies, those published as abstracts only, and unpublished data are eligible for inclusion. We will only include RCTs with supplemental oxygen during exercise training for COPD and exclude RCTs related to ambulatory oxygen or long-term oxygen therapy.

\section{Type of participants}

We will include participants 18 years of age or older, with a diagnosis of COPD diagnosed according to the GOLD criteria. ${ }^{1}$ We will exclude participants with other respiratory diseases.

\section{Type of intervention and comparators}

The active treatment group will receive supplemental oxygen during exercise training using wall oxygen units in a hospital or clinic, portable oxygen cylinders, liquid oxygen canisters, and oxygen concentrators.

The control group will be provided sham gas, such as compressed air, through a wall unit or cylinders or will receive no intervention under room air.

The exercise training performed in both groups is defined as a supervised or unsupervised inpatient, outpatient, home-based intervention that includes some form of exercise training applied to COPD patients.

\section{Types of outcome}

\section{Primary outcome}

1. Exercise capacity: 6-minute walking distance (6MWD: $\mathrm{m}$ ) or shuttle walking distance (SWT: $\mathrm{m}$ ) or other measures, e.g., peak oxygen consumption. (Peak $\mathrm{VO}_{2}: \mathrm{mL} / \mathrm{kg} / \mathrm{min}$ )

2. Maximum exercise load of exercise training, e.g., workload (Watts).

3. Dyspnea scores at the end of the exercise test, e.g. Borg score.

4. Health-related QOL assessed by questionnaires, e.g., 36-item Short Form Health Survey (SF-36).

\section{Search methods for identification of studies}

\section{Electronic searches}

This meta-analysis will be carried out according to the PRISMA Statement. ${ }^{19}$ We will conduct searches for studies with inception dates up to May 31, 2020 in the Cochrane Central Register of Controlled Trials (CEN-TRAL), MEDLINE, and EMBASE electronic databases. We will also search abstracts and meeting presentations utilizing the same search terms from Medical Subject Headings (MeSH). The search 
strategy will be based on discussions with an information specialist, and will be modified appropriately for each database. The search strategy includes a combination of free text words, words in titles/abstracts, and medical subject headings, such as "pulmonary disease, chronic obstructive," "supplemental oxygen," "oxygen inhalation therapy," and "exercise." Searches are limited to peer-reviewed research involving human participants. The detailed strategy and details of the formula that will be used to search the databases are shown in table 1.

\section{Searching other resources}

We will check the reference lists of all primary studies and review articles for additional references. Further, we will contact experts in the field to ask if they know of any ongoing or unpublished trials.

\section{Selection of studies}

Two review authors (SK and SY) will independently screen the titles and abstracts of all potentially relevant studies identified by the search, and code them as "retrieve" (eligible or potentially eligible/unclear) or "do not retrieve." In the case of disagreements, other review authors will be asked to arbitrate (KN and TY). SK and SY will retrieve the full text reports/publications, and independently screen the full texts and identified studies for inclusion. SK and SY will also identify and record reasons for the exclusion of ineligible studies. Any disagreements will be resolved by discussion or by consulting other review authors (KN and TY) when necessary. The review authors will identify and exclude duplicates, and collate multiple reports of the same study so that the unit of interest in the review will be each study, rather than each report. We will record the selection process in sufficient detail to complete a PRISMA flow diagram and "Characteristics of excluded studies" table. A data collection form piloted in at least one study in the review will be used to record study characteristics and outcome data. Two review authors (SK and SY) will extract characteristics from the included studies as follows:

1. Methods: study design, total duration of study, duration of follow-up, number of subjects, study setting, and date of study.

2. Participants: sample size, mean age, sex, history of smoking, history of oxygen therapy, forced expiratory volume in $1 \mathrm{~s}\left(\mathrm{FEV}_{1}\right)$ after bronchodilator administration, and arterial partial pressure of oxygen $\left(\mathrm{PaO}_{2}\right)$ at baseline.

3. Interventions: type of supplemental oxygen device, oxygen supplemental settings, such as continuous flow or demand delivery systems, and their flow rates, type of intervention as control, such as compressed air (and the flow rate), or room air, type of exercise training as frequency, duration, and contents.

4. Outcomes: exercise capacity, maximum exercise load of exercise training, dyspnea scores at the end of exercise test, health-related QOL assessed by questionnaire survey.

5. Notes: funding for trial and conflicts of interest of the trial authors.

\section{Data collection of studies}


Two review authors (SK and SY) will independently extract outcome data from the included studies and check each other's data extraction. One review author (SK) will transfer data into the Review Manager 5.3 file (RevMan 2019) and the EZR statistical program. ${ }^{23}$ SK and SY will double-check that data have been entered correctly by comparing the data presented in the systematic review with the study reports. A second review author (KN) will spot-check the study characteristics for accuracy against the trial report.

\section{Assessment of risk of bias}

Two review authors (SK and SY) will independently assess risk of bias for each study using the criteria outlined in the (RoB version 2) in the Cochrane Handbook for Systematic Reviews of Interventions. ${ }^{19}$ Any disagreements will be resolved by discussion, by involving other review authors ( $K N$ and $Y T$ ), or by contacting the authors of the included studies. We will assess risk of bias according to the following domains:

1. Bias arising from the randomization process

2. Bias due to deviations from intended interventions

3. Bias due to missing outcome data

4. Bias in measurement of the outcome

5. Bias in selection of the reported result

We will grade each potential source of bias as low risk, some concerns, or high risk and provide a quote from the study report together with a justification for our judgment in the "Risk of bias" table. We will summarize the risk of bias judgments across different studies for each of the domains listed.

\section{Measures of treatment effect}

We will analyze continuous data as the mean difference (MD) or standardized mean difference (SMD) with $95 \%$ confidence interval $(95 \% \mathrm{Cl})$. The MD is the absolute difference between the mean change before and after the intervention in a trial. The SMD is used as a summary statistic in the meta-analysis when the studies all assessed the same outcome but measured it in a variety of ways. We will enter data presented as a value with a consistent direction of effect. When the standard deviation (SD) for change from the baseline is not available, we will calculate the SD using Review Manager (RevMan) version 5.3. All Cls have two-sided probability coverage of $95 \%$. A $p$-value $<0.05$ will be considered significant. We will carry out sensitivity analysis when there is a high risk of bias that could affect the results.

\section{Assessment of heterogeneity}

We will assess statistical heterogeneity in each meta-analysis with $\mathrm{Tau}^{2}, \mathrm{I}^{2}$, and $\mathrm{Chi}^{2}$ statistics following the Cochrane Handbook for Systematic Reviews. ${ }^{19}$ We regard heterogeneity as substantial with $\mathrm{I}^{2}>0 \%$ and either the $\mathrm{Tau}^{2}$ is $>$ zero or $p<0.10$ in the $c^{2}$ test for heterogeneity. Data from each study will be 
pooled with fixed-effects modeling when there is no heterogeneity $\left(p>0.1,1^{2} \leq 40 \%\right)$. We will perform meta-analyses with random-effects models when there is heterogeneity $\left(p<0.1,1^{2}>40 \%\right)$.

\section{Subgroup analysis}

We plan to carry out the following subgroup analyses for primary outcomes if we obtain an $\mathrm{I}^{2}$ score > $50 \%$.

1. Severity of hypoxemia of rest at baseline $\left(\mathrm{PaO}_{2} \leq 60\right.$ Torr or $\mathrm{PaO}_{2}>60$ Torr $)$

2. Severity of exercise-induced desaturation at baseline (exercise induced desaturation: either decrease of $\mathrm{SpO}_{2} \geq 4 \%$ and nadir $\mathrm{SpO}_{2}$ during exercise $\leq 88 \%$ or not-exercise induced desaturation)

3. Severity of airflow limitation (\%FEV $1 \geq 50 \%$ or $\left.\% \mathrm{FEV}_{1}<50 \%\right)$

We plan to explore differences in outcomes in these subgroups if the number of collected studies is sufficient.

\section{Sensitivity analysis}

We plan to carry out sensitivity analysis for primary outcomes if the high risk of bias of some of the included studies affected the results. We will define "high risk" in each study as at least one domain evaluated as "high risk" or some domains are allocated as "some concern" that can decrease the reliability of the results. ${ }^{18}$ We plan to carry out the following sensitivity analyses.

\section{Meta-regression}

If there is any statistically heterogeneity or methodological heterogeneity, we will perform meta-regression using $\mathrm{PaO}_{2}$ at baseline (Torr) and decreases of $\mathrm{SpO}_{2}$ in exercise tests as covariates to investigate whether the severity of desaturation at baseline affects the effectiveness of exercise tolerance.

\section{Assessment of reporting biases}

When 10 or more studies are included in a meta-analysis, we will create a funnel plot and examine its asymmetry visually to explore any publication bias.

\section{Assessment of evidence in individual study}

We will use the GRADE criteria to assess the quality of evidence for each outcome according to four levels (high, moderate, low, or very low) ${ }^{21}$ The quality of evidence will be reduced by any one of the following limitations: risk of bias, inconsistency, indirectness, imprecision and publication bias. Two investigators (SK, SY) will independently conduct those assessment. Investigators will resolve disagreements between the two investigators through discussion, with a third reviewer available for adjudication (KN). 


\section{Discussion}

As exercise-induced desaturation is involved in adverse sequelae and higher risk of mortality in COPD, supplemental oxygen during exercise training has been prescribed in COPD patients who show severe desaturation. The meta-analysis in 2019 concluded that supplemental oxygen during exercise training does not further improve exercise tolerance compared to exercise training alone. However, especially in COPD patients with severe desaturation at rest and on exertion, supplemental oxygen during exercise training may be effective to improve exercise tolerance. A previous study reported that patients only who show exercise induced-desaturation in COPD, and who walked $10 \%$ or more further with ambulatory oxygen on shuttle walking test showed significant improvements in exercise tolerance by an exercise training program with supplemental oxygen. Therefore, we will conduct meta-regression analyses to examine the association between efficacy of exercise training with supplemental oxygen and severity of desaturation at baseline. However, the quantity and quality of the trials will possibly be the main limitation of this research.

\section{Abbreviatons}

COPD: chronic obstructive pulmonary disease

SMD: standardized mean difference

GRADE: grading of recommendations assessment, development, and evaluation

GOLD: global initiative for chronic obstructive lung disease

QOL: quality of life

PRISMA: preferred reporting items for systematic reviews, meta-analyses

RCT: randomized controlled trial

6MWD: 6-minute walking distance

SWT: shuttle walking distance

Peak $\mathrm{VO}_{2}$ : peak oxygen consumption

SF-36: 36-item short form health survey

CEN-TRAL: cochrane central register of controlled trials

MeSH: medical Subject Headings

$\mathrm{FEV}_{1}$ : forced expiratory volume in $1 \mathrm{~s}$ 
$\mathrm{PaO}_{2}$ : arterial partial pressure of oxygen

MD: mean difference

95\% Cl: 95\% confidence interval

\section{Declarations}

\section{Ethics approval and consent to participate}

Not applicable.

\section{Consent for publication}

Not applicable.

\section{Availability of data and materials}

The datasets generated during and/or analyzed during the current study are available from the corresponding author on reasonable request.

\section{Compenting interests}

The authors declare that they have no competing interests

\section{Funding}

This research received no specific grants from any funding agency in the public, commercial, or not-forprofit sectors.

\section{Authors' contributions}

SK and SY contributed to the conception of the study. KN, TY, SM and YS designed the methods of the systematic review and KF will supervise those design and analysis. All authors were involved in the critical revision, for the intellectual content, and read and approved the final manuscript.

\section{Acknowledgements}

Not applicable.

\section{References}

1. Global Initiative for Chronic Obstructive Lung Disease, Global strategy for the diagnosis, management and prevention of chronic obstructive pulmonary disease (2019 report), World Health 
Organization. 2019 (cited 2019 February 26) Available from:https://goldcopd.org/wpcontent/uploads/2018/11/GOLD-2019-v1.7-FINAL-14Nov2018-WMS.pdf.

2. Adeloye D, Chua S, Lee C, Basquill C, Papana A, Theodoratou E, Nair H, Gasevic D, Campbell H, Chan KY. Global and regional estimates of COPD prevalence: Systematic review and meta - J Glob Health. 2015: 5: 020415

3. Abubakar II, Tillmann T, Banerjee A. Global, regional, and national age-sex specific all-cause and cause-specific mortality for 240 causes of death, 1990-2013: a systematic analysis for the Global Burden of Disease Study 2013. Lancet. 2015: 385: 117-171.

4. McCarthy B, Casey D, Devane D, Murphy K, Murphy E, Lacasse Y. Pulmonary rehabilitation for chronic obstructive pulmonary disease. Cochrane Database Syst Rev. 2015: 2: CD003793.

5. Wagner PD, Dantzker DR, Dueck R, Clausen JL, West JB. Ventilation-perfusion inequality in chronic obstructive pulmonary disease. J clin invest. 1977: 59: 203-216.

6. Elbehairy AF, Ciavaglia CE, Webb KA, Guenette JA, Jensen D, Mourad SM, Neder JA, O'Donnell DE. Pulmonary gas exchange abnormalities in mild chronic obstructive pulmonary disease. Implications for dyspnea and exercise intolerance. Am J respir Crit Care Med. 2015: 191: 1384-1394.

7. O'Donnell DE, D'Arsigny C, Fitzpatrick M, Webb KA. Exercise hypercapnia in advanced chronic obstructive pulmonary disease: the role of lung hyperinflation. Am J Respir Crit Care Med. 2002: 166: 663-668.

8. Zafar MA, Tsuang W, Lach L, Eschenbacher W, Panos RJ. Dynamic hyperinflation correlates with exertional oxygen desaturation in patients with chronic obstructive pulmonary disease. Lung. 2013: 191: 177-182.

9. Kent BD, Mitchell PD, McNicholas WT. Hypoxemia in patients with COPD: cause, effects, and disease progression. Int J Chron Obstruct Pulmon Dis. 2011: 6: 199.

10. Casanova C, Cote C, Marin JM, Pinto-Plata V, de Torres JP, Aguirre-Jaíme A, Vassaux C, Celli BR. Distance and oxygen desaturation during the 6-min walk test as predictors of long-term mortality in patients with COPD. Chest. 2008: 134: 746-752.

11. Neunhäuserer D, Steidle-Kloc E, Weiss G, Kaiser B, Niederseer D, Hartl S, Tschentscher M. Egger A, Studnicka M. Supplemental oxygen during high-intensity exercise training in nonhypoxemic chronic obstructive pulmonary disease. Am J med. 2016: 129: 1185-1193.

12. Garrod R, Paul EA, Wedzicha JA. Supplemental oxygen during pulmonary rehabilitation in patients with COPD with exercise hypoxaemia. Thorax. 2000: 55: 539-543.

13. Wadell K, Henriksson-Larsén K, Lundgren R. Physical training with and without oxygen in patients with chronic obstructive pulmonary disease and exerciseinduced hypoxaemia. J Rehabil Med. 2001: 33: 200-205.

14. Alison JA, McKeough ZJ, Leung RW, Holland AE, Hill K, Morris NR, Jenkins S, Spencer LM, Hill CJ, Lee AL, Seale H, Cecins N, McDonald CF. Oxygen compared to air during exercise training in COPD with exercise-induced desaturation.Eur Respir J. 2019: 53: 1802429. 
15. Nonoyama M, Brooks D, Lacasse $\mathrm{Y}$, Guyatt GH, Goldstein RS. Oxygen therapy during exercise training in chronic obstructive pulmonary disease. Cochrane Database of Syst Rev.2007: 18: CD005372.

16. Liu Y, Gong F. Determination of whether supplemental oxygen therapy is beneficial during exercise training in patients with COPD: A systematic review and meta-analysis. Exp Ther Med. 2019: 18: 4081 -

17. Dyer F, Callaghan J, Cheema K, Bott J. Ambulatory oxygen improves the effectiveness of pulmonary rehabilitation in selected patients with chronic obstructive pulmonary disease. Chron Respir Dis. 2012: 9: 83-91.

18. Spielmanns M, Fuchs-Bergsma C, Winkler A, Fox G, Krüger S, Baum K. Effects of Oxygen Supply During Training on Subjects With COPD Who Are Normoxemic at Rest and During Exercise: A Blinded Randomized Controlled Trial. Respir Care. 2015: 60: 540 -

19. Higgins JPT, Thomas J, Chandler J, Cumpston M, Li T, Page MJ, Welch VA (editors). Cochrane Handbook for Systematic Reviews of Interventions version 6.0 (updated July 2019). Cochrane, 2019. Available from training.cochrane.org/handbook.

20. Moher David, Liberati Alessandro, Tetzlaff Jennifer, Altman Douglas G. Preferred reporting items for systematic reviews and meta-analyses: the PRISMA statement BMJ 2009; 339:b2535

21. Balshem H, Helfand M, Schünemann HJ, Oxman AD, Kunz R, Broxek J, Vist GE, Falck-Ytter Y, Meerpohi J, Norris S, Guyatt GH. GRADE guidelines: 3. Rating the quality of evidence. J Clin Epidemiol. 2011; 64: 401 -

22. Moher D, Shamseer L, Clarke M, Ghersi D, Liberati A, Petticrew M, Shekelle P, Stewart LA. Preferred Reporting Items for Systematic Review and Meta-Analysis Protocols (PRISMA-P) 2015 statement. Syst Rev. 2015: 4: 1.

23. Kanda Y. Investigation of the freely available easy-to-use software 'EZR' for medical statistics., Bone Marrow Transplant. 2013. 48: 452-458.

\section{Tables}

Table 1. The detailed strategy and details of formula for search in databases

(A). MEDLINE

1. Lung Diseases [MeSH Terms]

2. Lung Diseases, Obstructive [MeSH Terms]

3. Pulmonary Disease, Chronic Obstructive [MeSH Terms]

4. Bronchial Diseases [MeSH Terms]

5. Emphysema [MeSH Terms]

6. Hypoxia [MeSH Terms]

7. Hypoxemia [MeSH Terms] 
8. COPD [All Fields]

9. COAD [All Fields]

10. COBD [All Fields]

11. exercise induced-desaturation [Title/Abstract])

12. \#1OR \#2 OR \#3 OR \#4 OR \#5 OR \#6 OR \#7 OR \#8 OR \#9 OR \#10

13. supplemental oxygen [Title/Abstract]

14. oxygen Inhalation Therapy [MeSH Terms]

15. oxygen supplementation [Title/Abstract]

16. oxygen supply [Title/Abstract]

17. Oxygen [MeSH Terms]

18. \#13 OR \#14 OR \#15 OR \#16 OR \#17

19. exercise [MeSH Terms]

20. resistance Training [MeSH Terms]

21. exercise Therapy [MeSH Terms]

22. high-Intensity Interval Training [MeSH Terms]

23. physical Therapy Modalities [MeSH Terms]

24. physical Conditioning, Human [MeSH Terms]

25. rehabilitation [MeSH Terms]

26. \#19 OR \#20 OR \#21 OR \#22 OR \#23 OR \#24

27. randomized Controlled Trial [Title/Abstract]

28. trial [Title/Abstract]

29. randomized [Title/Abstract]

30. randomized [Title/Abstract]

31. randomly [Title/Abstract])

32. placebo [Title/Abstract]

33. \#27 OR \#28 OR \#29 OR \#30 OR \#31 OR \#32

34. Animals [Title/Abstract])

35. \#33 NOT \#34

36. \#12 AND \#18 AND \#26 AND \#35

(B). Embase

1. lung diseases

2. obstructive lung diseases 
3. chronic obstructive pulmonary disease

4. bronchial diseases

5. emphysema

6. hypoxia

7. hypoxemia

8. COPD: ab

9. exercise induced-desaturation

10. \#1 OR \#2 OR \#3 OR \#4 OR \#5 OR \#6 OR \#7 OR \#8 OR \#9

11. oxygen inhalation therapy

12. supplemental oxygen

13. oxygen supplementation

14. oxygen supply

15. \#11 OR \#12 OR \#13 OR \#14

16. exercise

17. resistance training

18. exercise therapy

19. interval training

20. physical therapy

21. rehabilitation

22. physical conditioning

23. exercise training

24. \#16 OR \#17 OR \#18 OR \#19 OR \#20 OR \#21 OR \#22 OR \#23

25. randomized controlled trial

26. $a b$

27. $a b$

28. $a b$

29. placebo

30. \#25 OR \#26 OR \#27 OR \#28 OR \#29

31. $a b$

32. \#30 NOT \#31

33. \#10 AND \#15 AND \#24 AND \#32

(C). Cochrane Central Register of Controlled Trials 
1. MeSH descriptor: [Lung Diseases] explode all trees

2. MeSH descriptor: [Lung Diseases, Obstructive] explode all trees

3. MeSH descriptor: [Pulmonary Disease, Chronic Obstructive] explode all trees

4. MeSH descriptor: [Bronchial Diseases] explode all trees

5. MeSH descriptor: [Emphysema] explode all trees

6. MeSH descriptor: [Hypoxia] explode all trees

7. Hypoxemia

8. COPD: ti,ab,kw OR COAD: ti,ab,kw OR COBD: ti,ab,kw OR exercise induced-desaturation: ti,ab,kw

9. \#1 OR \#2 OR \#3 OR \#4 OR \#5 OR \#6 OR \#7 OR \#8

10. MeSH descriptor: [Oxygen Inhalation Therapy] explode all trees

11. supplemental oxygen

12. oxygen supplementation

13. oxygen supply

14. MeSH descriptor: [Oxygenators] explode all trees

15. \#10 OR \#11 OR \#12 OR \#13 OR \#14

16. MeSH descriptor: [Exercise] explode all trees

17. MeSH descriptor: [Resistance Training] explode all trees

18. MeSH descriptor: [Exercise Therapy] explode all trees

19. MeSH descriptor: [High-Intensity Interval Training] explode all trees

20. MeSH descriptor: [Physical Therapy Modalities] explode all trees

21. MeSH descriptor: [Physical Conditioning, Human] explode all trees

22. Rehabilitation

23. \#16 OR \#17 OR \#18 OR \#19 OR \#20 OR \#21 OR \#22

24. Randomized Controlled Trial: pt OR trial: ti,ab,kw OR randomized: ti,ab,kw OR placebo: ti,ab,kw OR randomly: ti,ab,kw

25. animals

26. \#24 NOT \#25

27. \#9 AND \#15 AND \#23 AND\#26 